\title{
Calculating the Envelope Correlation Coefficient Directly From Spherical Modes Spectrum
}

\author{
Adam Narbudowicz \\ Technological University Dublin, adam.narbudowicz@mydit.ie \\ Max Ammann \\ Technological University Dublin, max.ammann@tudublin.ie \\ R. Cornelius \\ Institute of High Frequency Technology, Achen, Germany
}

See next page for additional authors

Follow this and additional works at: https://arrow.tudublin.ie/ahfrccon

Part of the Electrical and Computer Engineering Commons

\section{Recommended Citation}

Narbudowicz, A., Ammann, M. \& Cornelius, R. (2017). Calculating the envelope correlation co-efficient directly from spherical modes spectrum. 2017 11th European Conference on Antennas and Propagation (EUCAP). DOI: 10.23919/EuCAP.2017.7928132

This Conference Paper is brought to you for free and open access by the Antenna \& High Frequency Research Centre at ARROW@TU Dublin. It has been accepted for inclusion in Conference Papers by an authorized administrator of ARROW@TU Dublin. For more information, please contact arrow.admin@tudublin.ie, aisling.coyne@tudublin.ie,gerard.connolly@tudublin.ie.

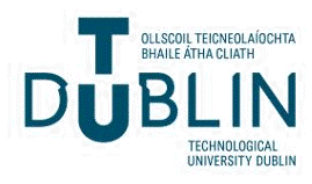


Authors

Adam Narbudowicz, Max Ammann, R. Cornelius, and D. Heberling

This conference paper is available at ARROW@TU Dublin: https://arrow.tudublin.ie/ahfrccon/42 


\title{
Calculating the Envelope Correlation Coefficient Directly From Spherical Modes Spectrum
}

\author{
Rasmus Cornelius ${ }^{1}$, Adam Narbudowicz ${ }^{2}$, Max J. Ammann ${ }^{2}$ and Dirk Heberling ${ }^{1}$ \\ ${ }^{1}$ Institute of High Frequency Technology, RWTH Aachen University, Aachen, Germany \\ ${ }^{2}$ Antenna \& High Frequency Research Centre, Dublin Institute of Technology, Dublin, Ireland
}

\begin{abstract}
This paper proposes an improved technique to calculate the Envelope Correlation Coefficient (ECC) by directly using the spherical mode spectrum of the antenna under test. The technique avoids errors due to numerical pattern integration and simplifies post-processing for nearfield measurements. The technique is successfully tested on two different antenna types, in the total number of four MIMO configurations.
\end{abstract}

Index Terms-MIMO, envelope correlation coefficient, spherical near field, antenna measurement.

\section{INTRODUCTION}

The constantly growing need for higher wireless data throughput has resulted in common use of MIMO technology across the globe. To support this trend there is a great and increasing number of MIMO antenna designs, many of which are incorporated into a small volume [1-4]. Such antennas need to be characterized with respect to their performance within MIMO systems and considering often complex interactions between multiple antenna ports.

The Envelope Correlation Coefficient (ECC) has become a standard parameter for MIMO antennas and is defined as:

$\rho_{\mathrm{ECC}}=\frac{\iint_{4 \pi} \boldsymbol{E}_{1}(\theta, \phi) \cdot \boldsymbol{E}_{2}^{*}(\theta, \phi) d \Omega}{\sqrt{\iint_{4 \pi} \boldsymbol{E}_{1}(\theta, \phi) \cdot \boldsymbol{E}_{1}^{*}(\theta, \phi) d \Omega \iint_{4 \pi} \boldsymbol{E}_{2}(\theta, \phi) \cdot \boldsymbol{E}_{2}^{*}(\theta, \phi) d \Omega}}$

where $\boldsymbol{E}_{1}$ and $\boldsymbol{E}_{2}$ are far-field radiation patterns, generated from ports 1 and 2 respectively. The metric can be seen as a correlation of two radiation patterns, which, under the assumption that the incoming signals are isotropically distributed, yields also the correlation of MIMO signals at receiver inputs [5]. The lower the correlation, the higher data throughput can be supported by the MIMO antenna. To calculate (1), one needs to perform a full sphere antenna measurement for each port.

Hallbjorner in [6] has proposed a simplified approach to approximate the ECC when only S-parameters and efficiencies $\eta_{1}$ and $\eta_{2}$ are known:

$\rho_{\mathrm{ECC}}=-\frac{S_{11} S_{12}^{*}+S_{21} S_{22}^{*}}{\sqrt{\left(1-\left|S_{11}\right|^{2}-\left|S_{21}\right|^{2}\right)\left(1-\left|S_{22}\right|^{2}-\left|S_{12}\right|^{2}\right) \eta_{1} \eta_{2}}}$

This formula is valid if the losses between both antennas are either independent or if the loss correlation is small enough to be neglected. Although measurement of S-parameters is straightforward, measurement of efficiencies poses certain challenges. A technique to measure efficiencies of MIMO antennas with Wheeler's Cap has been recently proposed by Moharram and Kishk in [7] but provides only limited accuracy. In most cases, measurements in an anechoic chamber are required for accurate estimation of efficiencies, thus limiting the benefits of (2). Please also note that neglecting the efficiency term in (2) will lead to a large error in the calculated ECC [8]. Thus to determine the ECC an anechoic chamber or reverberation chamber is required.

Spherical Near-Field (SNF) measurements are recently becoming more popular, especially for characterization of small antennas. Rather than directly sampling far-field radiation patterns, the technique calculates spherical modes of the antenna under test from near-field measurements [9]. The far-field radiation pattern is then calculated from the modal spectrum. The number of spherical modes is proportional to $\left(r_{\mathrm{AUT}} / \lambda\right)^{2}$, where $r_{\mathrm{AUT}}$ is a radius of a sphere that fully encloses the Antenna Under Test (AUT).

In this paper we demonstrate, that the ECC can be calculated directly from the spherical mode spectrum of an antenna, without the need for far-field patterns. This approach requires less post-processing and offers greater precision, as it avoids the error when the radiation pattern is numerically integrated over a sphere. Furthermore, it provides a more intuitive understanding of the relation between antenna size and possible number of uncorrelated data streams.

\section{PRINCIPLES}

The spherical far-field antenna radiation pattern can be described as a superposition of spherical modes:

$\boldsymbol{E}(\theta, \phi)=k \sqrt{Z_{0}} \sum_{l=1}^{\infty} \sum_{m=-l}^{l} \sum_{s=1}^{2} q_{s m l} \boldsymbol{K}_{s m l}^{(c)}(\theta, \phi)$

where $\boldsymbol{K}_{s m l}$ denotes the spherical harmonics, $q_{s m l}$ its complex coefficient, $k$ is the wavenumber and $Z_{0}$ is the impedance of free space. The order $m(-l \leq m \leq l)$ of the spherical harmonics describes the azimuthal variation $(\phi)$ of the field. The variation in $\theta$ depends on the degree $l$ and the order $m$. The index $s \in\{1,2\}$ is connected with the components of transversal electric (TE) and transversal magnetic (TM) waves. The upper index (c) specifies the radial function of the spherical wave [9]. 
By definition the modes are orthogonal to each other, thus their correlation can be expressed using Dirac's delta:

$\iint_{4 \pi} \boldsymbol{K}_{s m l}^{(3)}(\theta, \phi) \cdot \boldsymbol{K}_{\sigma \mu v}^{(4)}(\theta, \phi) d \Omega=\delta_{s \sigma} \delta_{m,-\mu} \delta_{l v}(-1)^{m}$

with

$$
\begin{aligned}
\left(\sum_{v=1}^{\infty} \sum_{\mu=-v}^{v} \sum_{\sigma=1}^{2} q_{\sigma \mu v}\right. & \left.\boldsymbol{K}_{\sigma \mu \nu}^{(3)}(\theta, \phi)\right)^{*} \\
& =\sum_{v=1}^{\infty} \sum_{\mu=-v}^{v} \sum_{\sigma=1}^{2}(-1)^{\mu}\left(q_{\sigma,-\mu, v}\right)^{*} \boldsymbol{K}_{\sigma \mu v}^{(4)}(\theta, \phi)
\end{aligned}
$$

and by combining (4) and (3) into (1) we obtain:

$$
=\frac{\rho_{\mathrm{ECC}}}{\sqrt{\left(\sum_{l=1}^{\infty} \sum_{m=-l}^{l} \sum_{s=1}^{2} q_{s m l}^{1}\left(q_{s m l}^{1}\right)^{*}\right)\left(\sum_{l=1}^{\infty} \sum_{m=-l}^{l} \sum_{s=1}^{2} q_{s m l}^{2}\left(q_{s m l}^{2}\right)^{*}\right)}}
$$

This allows the calculation of the ECC directly from the mode spectrum, i.e. the coefficients $q_{s m l}$. Please note, that the field of each mode $\boldsymbol{K}_{s m l}$ is irrelevant and only the coefficients contribute to the ECC value.

\section{MEASUREMENTS AND DISCUSSION}

Eq. (5) was tested with measured data from three twoelement Printed Inverted-F Antennas (PIFA) and one dualmode patch antenna. The measurements were performed in spherical near-field antenna measurement facility of the Institute of High Frequency Technology, RWTH Aachen University.

\section{A. Printed Inverted F Antennas}

A simple PIFA antenna was designed in [10] to operate in the bandwidth of $2.45-2.6 \mathrm{GHz}$. To validate (5) three different configurations were prepared as a testbed, each consisting of two PIFAs. The configurations varied both orientation and distance between antennas in order to provide representative span of possible ECC values. This involved an opposite configuration, with antennas placed back-to-back at a distance of $48 \mathrm{~mm}$ (Fig. 1a); an orthogonal configuration with $30 \mathrm{~mm}$ spacing between antenna feeds (Fig. 1b); and parallel configuration with $20 \mathrm{~mm}$ spacing between antenna

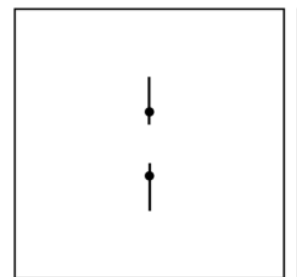

a)

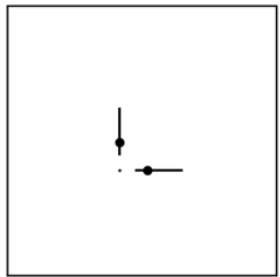

b)

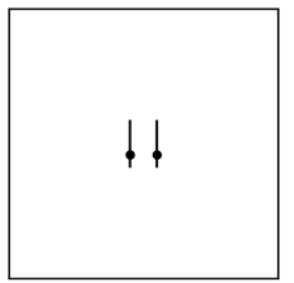

c)
Fig. 1. Three various PIFA configuration used as a testbed:

a) opposite with $48 \mathrm{~mm}$ distance between feeds;

b) orthogonal with $30 \mathrm{~mm}$ distance between feeds;

c) parallel with $20 \mathrm{~mm}$ distance between feeds.

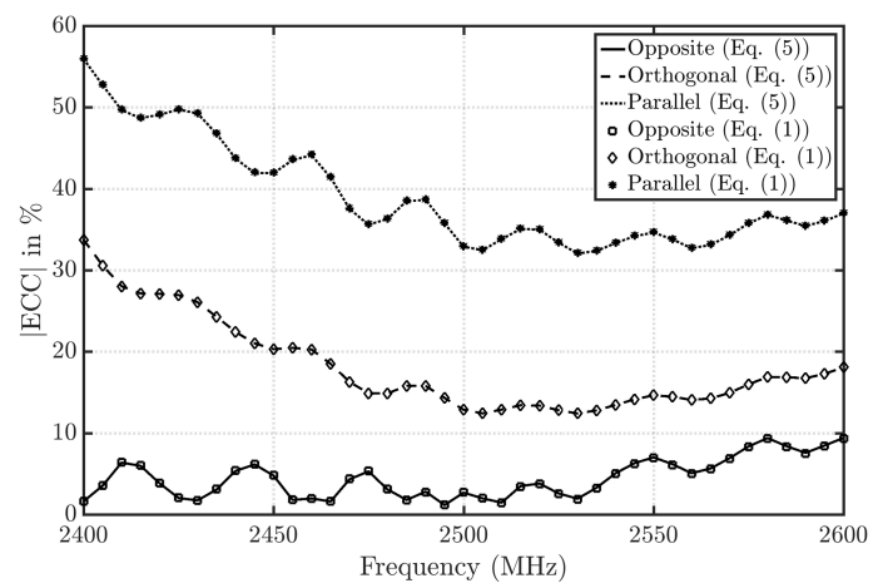

Fig. 2. ECC values calculated from (5) and (1) for the three investigated PIFA configurations.

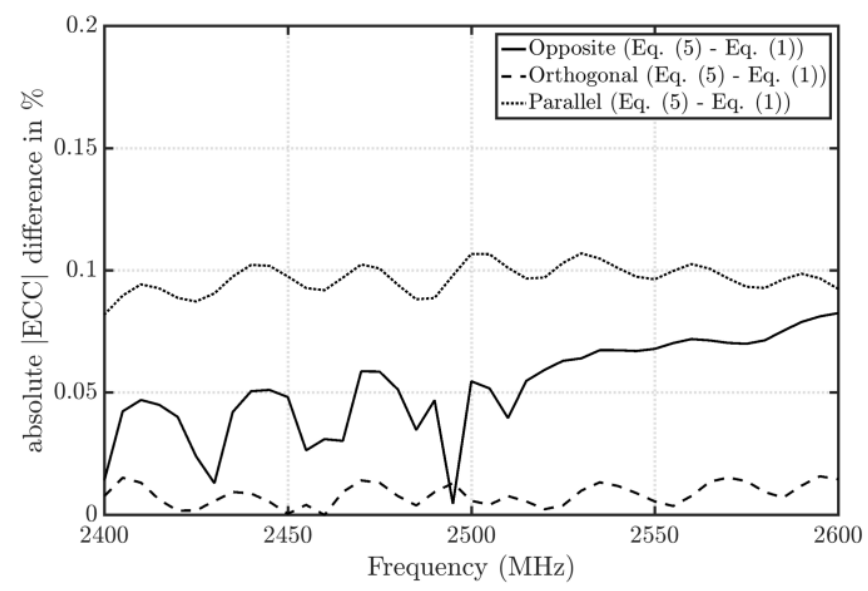

Fig. 3. Absolute ECC differences calculated by (5) and (1) for the three investigated PIFA configurations.

feeds (Fig. 1c). The antennas protruded from a square copper ground plane of $200 \times 200 \mathrm{~mm}$. This size was chosen in order to minimize the effect of the antenna mounting structure in the anechoic chamber.

The antennas were measured in the band from 2.4 to $2.6 \mathrm{GHz}$ with an angular resolution of $7.2^{\circ}$. The far-field was calculated with a resolution of 1 degree. The ECC values were calculated by (1) and (5). A very good overlap between both techniques can be seen in Fig. 2 . The absolute differences (Fig. 3) are almost for all configuration below $0.1 \%$.

\section{B. Multimode patch}

Multimode patch antennas have recently grown in popularity, as they offer a MIMO performance within a costefficient, low profile structure [3-4]. The antenna used as a testbed operates at $5.05 \mathrm{GHz}$ and consists of two stacked patches, operating in $\mathrm{TM}_{10}$ and $\mathrm{TM}_{40}$ mode. Fig. 4 shows the ECC values calculated with (1) and (5) and again the curves are very close to each other. 


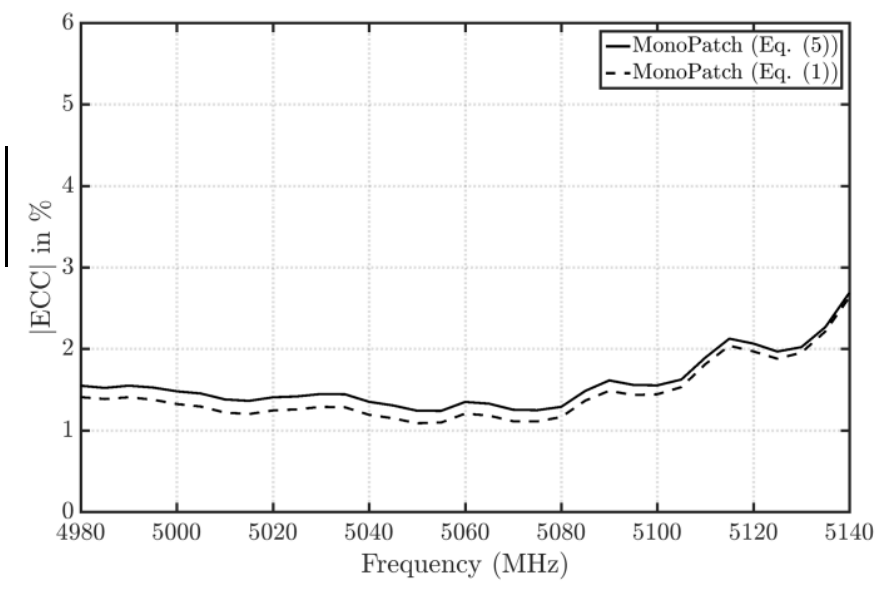

Fig. 4. ECC values calculated by (5) and (1) for the multimode patch antenna.

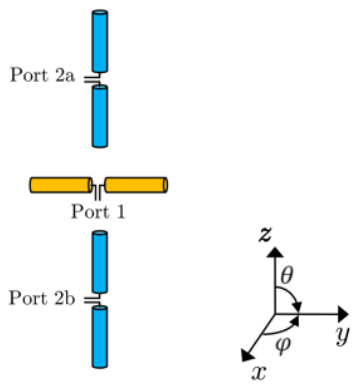

Fig. 5. Simulated antenna configuration.

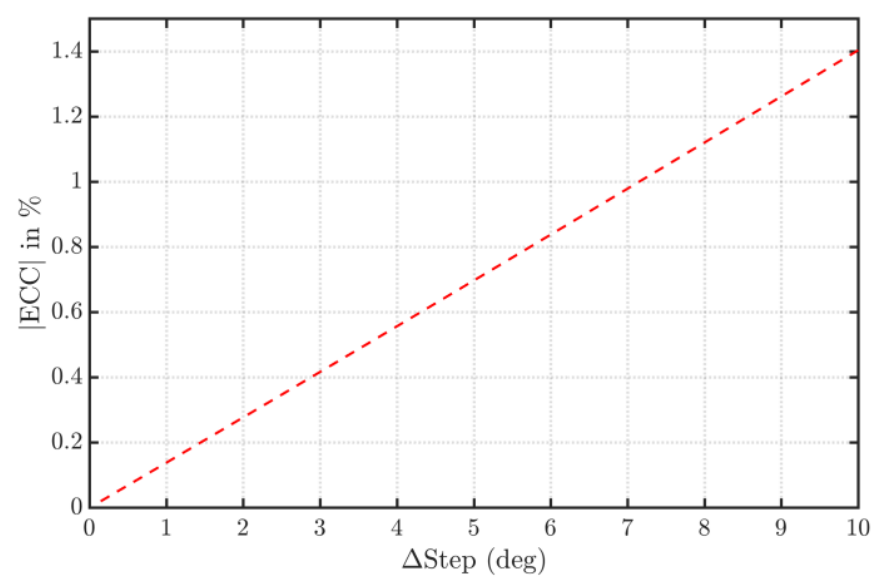

Fig. 6. ECC according to (1) as a function of angular resolution for the simulated antenna configuration.

\section{Simulated antenna configuration}

The ECC differences for the examples are caused by a numerical integration error when using standard trapezoidal method. We used the trapz function from Matlab for the spherical numerical integration via the trapezoidal method. In order to investigate this error in more details a perfect uncorrelated $(\mathrm{ECC}=0)$ antenna configuration was simulated. The antenna consist of a horizontal Hertzian dipole (port 1) and two vertical Hertzian dipoles port 2a/b separated by $\lambda / 2$ (see Fig. 5). Port $2 b$ has a $180^{\circ}$ phaseshift compared to port $2 \mathrm{a}$ creating a null in the xy-plane. The radiated modes are listed in table 1 and the radiation pattern should be perfectly uncorrelated. However, the calculated ECC according to (1) is affected by numerical errors and approaches zero with increasing angular resolution (see Fig. 6). Please note, that this error will change, depending on the spherical modes excited by each antenna and differs with antenna topology, as seen in Sections III.A and III.B. Calculation of the ECC based on the spherical mode spectrum does not have this dependency and is thus not affected by the numerical integration error.

TABLE I. $\quad\left|q_{\text {sml }}\right|$ OF SimULATEd ANTENNA CONFIGURATION

\begin{tabular}{|c|c|c|c|}
\hline \multicolumn{2}{|c|}{ Port 1 } & \multicolumn{2}{c|}{ Port 2 } \\
\hline Mode $(s, m, l)$ & $\left|q_{\text {slm }}\right|$ & Mode $(s, m, l)$ & $\left|q_{\text {slm }}\right|$ \\
\hline$(2,-1,1)$ & $\sqrt{2}$ & $(2,0,2)$ & 0.885 \\
\hline$(2,+1,1)$ & $\sqrt{2}$ & $(2,0,4)$ & 0.461 \\
\hline & & $(2,0,6)$ & 0.063 \\
\hline & & $(2,0,8)$ & 0.004 \\
\hline & & $(2,0,10)$ & $<0.001$ \\
\hline
\end{tabular}

\section{CONCLUSIONS}

The paper introduces a method to calculate the Envelope Correlation Coefficient directly from the spherical mode spectrum, without the need to extract the radiation pattern. The method was validated with four exemplary antennas. Furthermore it was demonstrated, that the proposed technique avoids the typical numerical integration error.

\section{ACKNOWLEDGMENT}

This work was supported by Irish Research Council under "ELEVATE: Irish Research Council International Career Development Fellowship - co-funded by Marie Cure Actions", grant no. ELEVATEPD/2014/79.

\section{REFERENCES}

[1] M. S. Sharawi, "Printed MIMO antenna systems: performance metrics, implementations and challenges," Forum for Electromagnetic Research Methods and Application Technologies (FERMAT), Vol. 1, 2014.

[2] D. Manteuffel, and R. Martens, "Compact multimode multielement antenna for indoor UWB massive MIMO," IEEE Transactions on Antennas and Propagation, vol. 64, issue 7, pp. 2689-2697, July 2016.

[3] A. Narbudowicz, S. Koziel, M. J. Ammann, and D. Heberling, "Planar dual-mode MIMO antenna with enhanced bandwidth," In Proc.: IEEE International Symposium on Antennas and Propagation \& USNC/URSI National Radio Science Meeting, Vancouver, Canada, pp. 703-704, July 2015

[4] N. Herscovici, C. Christodoulou, E. R. Iglesias, O. Q. Teruel, and M. S. Ferflifldez, "Compact multimode patch antennas for MIMO applications," IEEE Antennas Propag. Mag., vol. 50, no. 2, pp. $197-$ 205, Apr. 2008.

[5] P. S. Kildal, Foundations of Antenna Engineering: A Unified Approach for Line-of-sight and Multipath, Artech House Publishers, 2015.

[6] P. Hallbjorner, "The significance of radiation efficiencies when using S-Parameters to calculate the received signal correlation from two antennas," IEEE Antennas and Wireless Propagation Letters, vol. 4, pp. $97-99,2005$. 
[7] M. A. Moharram, and A. A. Kishk, "MIMO antennas efficiency measurement using wheeler caps," IEEE Transactions on Antennas and Propagation, vol. 64, issue 3, pp. 1115 - 1120, Mar. 2016.

[8] A. Narbudowicz, M. J. Ammann, and D. Heberling, "Impact of lossy feed on S-parameter based envelope correlation coefficient," In Proc.: 10th European Conference on Antennas and Propagation (EuCAP), Davos, Switzerland, April 2016.

[9] J. E. Hansen, Spherical Near-Field Antenna Measurements, P. Peregrinus, 1988.

[10] F. Reher, "Study of wheeler cap method for multiport MIMO antennas," B.Sc. thesis, Institute of High Frequency Technology, RWTH Aachen University, Aachen, Germany, 2016. 